\title{
TAp63alpha Induced Apoptosis Inhibited by Kaposi’s Sarcoma Herpesvirus Latency Nuclear Antigen
}

\author{
Suchitra Mohanty ${ }^{1 \#}$, Sushil Kumar Sahu ${ }^{1 \#}$, Nabanita Roy Chattopadhyay ${ }^{2 \#}$, Amit Kumar ${ }^{1}$, Piyanki Das ${ }^{2}$ and Tathagata Choudhuri $^{12^{*}}$ \\ ${ }^{1}$ Division of Infectious Disease Biology, Institute of Life Sciences, Bhubaneswar, India \\ ${ }^{2}$ Department of Biotechnology, Siksha Vhabana, Visva Bharati, Santiniketan, Bolpur, India \\ \#equally contributed
}

*Corresponding author: Tathagata Choudhuri, Department of Biotechnology, Siksha Vhabana, Visva Bharati, Santiniketan, Bolpur, India, Tel: 0674-2300728; Fax: (0674) 2300728; E-mail: tathagata.choudhuri@visva-bharati.ac.in

Received date: March 02, 2015, Accepted date: March 27, 2015, Published date: April 02, 2015

Copyright: @ 2015 Choudhuri T, et al. This is an open-access article distributed under the terms of the Creative Commons Attribution License, which permits unrestricted use, distribution, and reproduction in any medium, provided the original author and source are credited.

\begin{abstract}
Kaposi's sarcoma-associated herpesvirus (KSHV) infects a variety of human cells including cells of epithelial, mesenchymal and endothelial origin. The latency associated nuclear antigen (LANA) of this virus regulates the transcription of a number of viral and cellular genes essential for the survival of the virus in the host cell. TAp63a can induce apoptosis in stressed cells by upregulating various death receptors and loss of mitochondrial membrane potential. The present study demonstrates that LANA inhibits TAp63a-mediated apoptosis by a direct interaction with each other. This interaction also results in a reduction in loss of mitochondrial membrane potential caused by TAp63a. Therefore the present study indicates a possible mechanism of KSHV-infected cells to escape apoptosis and facilitate survival.
\end{abstract}

\section{Keywords: LANA; TAp63; KSHV; Apoptosis}

\section{Introduction}

Kaposi's sarcoma-associated herpesvirus (KSHV) or human herpesvirus-8, a member of the gammaherpesvirus family, causes mostly Kaposi's sarcoma (KS), primary effusion lymphoma (PEL), and multicentric Castleman's disease (MCD) [1-3]. It is recognized as a tumor virus but the complete mechanisms behind its tumorigenic effects remains to be explored. Infection from this virus cannot transform the primary endothelial cells but extends its survival, induces angiogenesis and causes chromosomal instability. KSHV is not capable of transforming mature B-cells also, but is required for the survival of PEL cell lines which are derived from B-cell lymphoma. In latently infected cells latency-associated nuclear antigen-1 (LANA) is required for viral replication and segregation of the viral genome during mitosis by tethering the viral genome to cellular chromatin. Moreover, LANA interacts with many cellular proteins, including the tumor suppressors p53 and pRb, and regulates the expression of several cellular and viral genes [4-9].

p63 and p73 are two homologs of p53 and so all these three tumor proteins belong to the same protein family. Although p53 is a wellknown tumor suppressor protein in mammalian cells, the roles of $\mathrm{p} 63$ and p73 are more diverse and include regulation of the cell cycle, senescence, apoptosis and an involvement in neuronal development [10-13]. All these three proteins share similar protein architecture with some overlapping functions, and all have dual gene structures generating multiple isoforms. In p63 and p73, internal promoters lead to the expression of transcriptionally active as well as $\mathrm{N}$-terminally truncated $(\Delta \mathrm{N})$ isoforms that lack the transactivating (TA) domain and act as dominant negative regulators of p53 and of p63 or p73 isoforms possessing the TA domain [10,14]. Among nearly 200 LANA-regulated cellular genes, seven are related to p53 signalling, and the key regulator is mouse double minute-2 (MDM2), promoting ubiquitination and subsequent degradation of p53 [15-18]. In contrast, MDM2 associates with p63 and inhibits its transcriptional activity by preventing its acetylation by $\mathrm{p} 300 / \mathrm{CPB}$ and subsequent activation [19-20].

Many viral antigens antagonize p53 and/or p63 in human cancers; examples include adenovirus E1B55K, SV40 large T-antigen, Tax 23 of HTLV-1 and the E6 and E7 proteins of HPV [21-26]. Unlike in the majority of all cancers, p53 mutations are infrequent in KSHVassociated malignancies, and the role of p53 in KSHV-associated pathogenesis appears to be complex. LANA interacts directly with p53 and represses p53-mediated transcriptional activity. Furthermore, p53 was reported to be ubiquitinated and degraded in the presence of LANA. However, p53-mediated DNA damage signalling was reported to be intact in PEL cells and activation of the p53 pathway using the MDM2 small-molecule inhibitor Nutlin-3 caused specific activation of apoptosis in PEL cells with wild-type p53. In PEL cells, LANA exists in a complex with $\mathrm{p} 53$ and MDM2, which disintegrates upon activation of p53 by DNA damage or treatment with the MDM2 inhibitor Nutlin-3, thereby restoring the normal function of the p53 tumour suppressor protein [5,27-30]. Here we investigate if LANA affects the p53 family member p63 and we report that LANA interacts with TAp63 isoforms and stabilizes them post-transcriptionally.

\section{Materials and Methods}

\section{Cell culture}

The adherent cell lines Saos-2 were grown in Dulbecco's modified eagle medium (cat no.P04-20350). Growth mediums (Pan Biotech, $\mathrm{GmbH}$, Germany) were supplemented with $10 \%$ foetal bovine serum (Australian origin), $20 \mathrm{unit} / \mathrm{ml}$ penicillin, and $100 \mu \mathrm{g} / \mathrm{ml}$ streptomycin (Sigma). Cultures were maintained in a humidified incubator at $37^{\circ} \mathrm{C}$ and $5 \% \mathrm{CO}_{2}$. 


\section{Plasmids}

PA3M-LANA constructs encoding full-length LANA was obtained from Erle S. Robertson, University of Pennsylvania, USA. The PCR amplified p63 fragments were cloned in-frame with a Flag epitope. (Gift from Gerry Melino, University of Rome, Italy).

\section{Antibodies}

The monoclonal antibodies mouse anti-myc (9E10), Mouse, rabbit or goat antibodies to p63, Flag and $\beta$-actin were purchased from Santa Cruz Biotechnology, Cell Signaling Technology or Imgenex India Ltd. HRP conjugated mouse or rabbit polyclonal secondary antibodies were purchased from above mentioned companies. Alexa-488 and 594 conjugated secondary antibodies used in immunofluorescence were purchased from Invitrogen.

Transfection: Transient transfection of expression vector was done with LipofectAMINE Plus reagent according to the manufacturer's instructions (Invitrogen Inc) or by electroporation using a BioRad Gene Pulser II electroporator. Ten million cells were collected, washed once in phosphate buffered saline (PBS) and then resuspended in 400 $\mu \mathrm{l}$ of either DMEM or RPMI 1640 according to the cell type. Once resuspended, the cells were transferred to $0.4 \mathrm{~cm}$ electroporation cuvettes and electroporated. Following electroporation the cells were plated in $10 \mathrm{ml}$ of supplemented media and grown at $37^{\circ} \mathrm{C}$ with $5 \%$ $\mathrm{CO}_{2}$ for 24 hours before harvesting. Transfections of siRNA were performed according to the manufacturer's instructions (Invitrogen Inc).

Immunoprecipitation and western blotting: Transfected cells were harvested, washed in ice-cold phosphate buffered saline (PBS) and lysed in $0.5 \mathrm{ml}$ ice-cold radioimmunoprecipitation assay (RIPA) buffer [0.5\% NP-40, $10 \mathrm{mM}$ Tris pH 7.5, $2 \mathrm{mM}$ EDTA, $150 \mathrm{mM} \mathrm{NaCl}]$ supplemented with protease inhibitor from Roche. Cellular debris was removed by centrifugation $\left(21000 \mathrm{~g}, 10 \mathrm{~min}, 4^{\circ} \mathrm{C}\right)$ and the supernatant was transferred to a fresh tube. Approximately $5 \%$ of the lysate was saved as an input control. Lysates were then precleared with isotype control and then rotated with $30 \mu \mathrm{l}$ of a 1:1 mixture of protein A- and protein G-conjugated Sepharose beads for $1 \mathrm{~h}$, at $4^{\circ} \mathrm{C}$. Beads were spun out and the supernatant was transferred to a fresh microcentrifuge tube and approximately $5 \%$ of the lysate was saved for input control. The protein of interest was captured by rotating overnight the remaining lysate with $1 \mu \mathrm{g}$ of specific antibody at $4^{\circ} \mathrm{C}$. Complexes were precipitated with $30 \mu \mathrm{l}$ of 1:1 mixture of protein A- and protein GSepharose beads. The samples were washed three times with ice-cold RIPA buffer, fractionated by SDS-PAGE and transferred onto a 0.45 $\mu \mathrm{m}$ nitrocellulose membrane for western blotting. The membranes were probed with the appropriate primary antibodies followed by incubation with appropriate HRP-tagged secondary antibodies. Then the membrane was exposed to luminal reagent of Santa Cruz Biotechnology and developed on X-ray film.

Immunofluorescence: Cells were grown onto slides and fixed using a 1:1 mixture of acetone and methanol. After fixation cells were washed three times in PBS and incubated in blocking buffer (PBS supplemented with $0.1 \%$ Triton $\mathrm{X} 100$ and 3\% BSA) at room temperature (RT) for $30 \mathrm{~min}$. Primary antibodies were diluted in blocking buffer and incubated with fixed cells for $1 \mathrm{~h}$ at RT. Cover slips containing cells were washed three times with PBS and incubated with appropriate secondary antibodies (1:2000) for $1 \mathrm{~h}$ at RT followed by three times washes with PBS. Secondary antibodies used were goat anti-human antibody Alexa Fluor 488 and goat anti-mouse antibody
Alexa Fluor 594 from Invitrogen. The last wash contained 4',6'diamidino-2-phenylindole (DAPI) for nuclear staining. Cover slips were then washed in PBS and mounted using Prolong anti-fade. Fluorescence was viewed by confocal microscopy.

Flow cytometry: Cells were cultured in 6-well culture plates. Cells were treated or not for 24 hours with doxorubicin at $5 \mu \mathrm{M}$ final concentration and incubated for additional 24 hours. The presence of apoptotic cells was detected by flow cytometry using propidium iodide and FITC conjugated Annexin V double labelling according to manufacturer's instructions (BD Bioscience). Similarly, apoptosis in transfected cells with different plasmid constructs were also determined. For the assessment of mitochondrial transmembrane potential, cells were incubated with doxorubicin for 24 hours and then for an additional $15 \mathrm{~min}$ at $37^{\circ} \mathrm{C}$ in the dark with $40 \mathrm{~nm} \mathrm{DiOC}$. Ten thousand cells per sample were analyzed flow cytometrically for $\mathrm{DiOC}_{6}$ fluorescence .

Statistical analysis: Graph Pad Prism (version 5.01) was used to perform statistical analyses. Difference between means was analyzed by ANOVA. A probability ( $p$ ) value of $<0.05$ was considered to reveal a significant difference. Data obtained in three separate experiments were statistically analyzed by either 1way ANOVA and 2way ANOVA followed by Tukey post-test and Boneferroni post-test respectively according to the experiment. ${ }^{* *} \mathrm{P}<0.001,{ }^{* *} \mathrm{P}<0.01,{ }^{\star} \mathrm{P}<0.05, \Delta \mathrm{P}>0.05$.

\section{Results}

\section{Flow cytometric analyses of cells treated with doxorubicin}

Cells were treated with doxorubicin for 24 hours and were analyzed for apoptosis. Cells were stained with AnnexinV-FITC and Propidium iodide for apoptosis analysis by FACS caliber. Control shows less than $5 \%$ cell death after 12 and 24 hours and a normal cell cycle distribution pattern. TAp63a-transfected cells show near about 20\% apoptosis whereas the cells transfected with TAp63a-LANA constructs show much less apoptosis (about 10\%) (Figure 1).

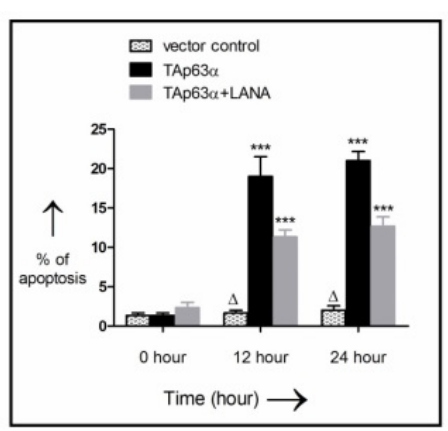

Figure 1: TAp63a induced apoptosis inhibited by LANA. TAp63a induces apoptosis of doxorubicin-treated cells within 12 and $24 \mathrm{hrs}$. Cells co-transfected with TAp63a and LANA shows less apoptosis throughout the study period, indicating a significant increase in viability of the cells by the presence of LANA ${ }^{* * *} \mathrm{P}<0.001$, $\left.{ }^{*} \mathrm{P}<0.01,{ }^{*} \mathrm{P}<0.05, \Delta \mathrm{P}>0.05\right)$. 
Citation: Mohanty S, Sahu SK, Chattopadhyay NR, Kumar A, Choudhuri T, et al. (2015) TAp63alpha Induced Apoptosis Inhibited by Kaposi's Sarcoma Herpesvirus Latency Nuclear Antigen. J Carcinog Mutagen 6: 221. doi:10.4172/2157-2518.1000221

Page 3 of 5

\section{Formation of bands in immunoprecipitation assays}

Immunoprecipitation experiments were done in doxorubicintreated cells transfected with Myc-tagged LANA and Flag-tagged TAp63a (Figure 2). Immunoprecipitation done with anti-Myc antibody shows clear bands for Myc-tagged LANA transfected cells (Figure 2. panel a). Similar bands are seen in immunoprecipitation done with anti-Flag antibody in Flag-tagged TAp63a transfected cells (Figure 2. panel b).

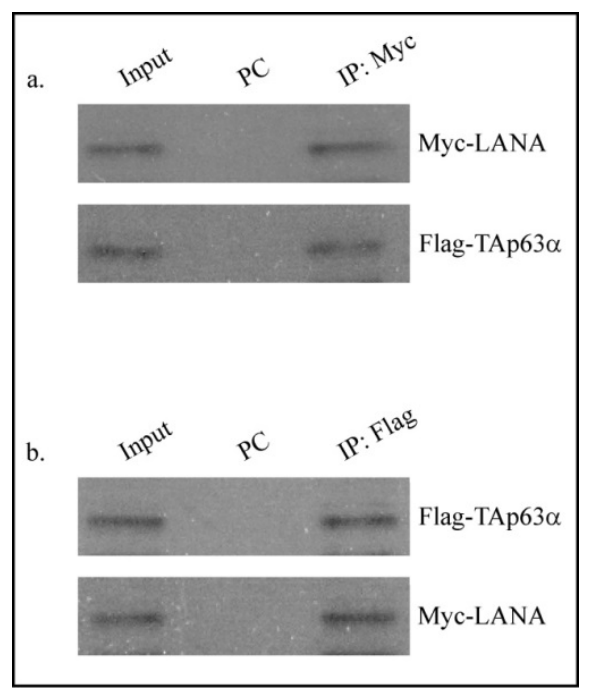

Figure 2: LANA forms a stable complex with p63. Cells transfected either with Myc-tagged LANA or with Flag-tagged TAp63 show similar bands in immunoprecipitation studies. a. Bands produced by Anti-Myc antibody and b. bands produced by Anti-Flag antibody. These data are the representative of three independent experiments, suggesting an interaction between TAp63 $\alpha$ and LANA in the doxorubicin treated cells.

\section{Co-localization study for LANA and p63}

Co-localization studies for TAp63a and LANA were done in the doxorubicin treated cells to further validate the interaction between them (Figure 3). The cell nuclei were counterstained with DAPI for demonstrating positional simulation. Flag-TAp63a was detected using Flag antibody, followed by Alexa Fluor 594 (red) and Myc-LANA was detected using Myc antibody followed by Alexa Fluor 488 (green). The merged image shows co-localization of TAp63 $\alpha$ and LANA.

\section{Assessment of loss of mitochondrial membrane potential}

Loss of mitochondrian membrane potential ( $\triangle \Psi \mathrm{mMMP})$ in TAp63a-mediated apoptosis is reported previously [30-32]. To determine any reduction in such loss of MMP in cells possessing LANA, DiOC6 fluorescence analysis was done as described earlier [33]. Cells transfected with TAp63a alone and with both TAp63aand LANA were analyzed flow cytometrically (Figure 4). As a positive control of apoptosis, untreated cells (UC) which were treated with doxorubicin only are used. Another group of Saos-2 cells (SC) with no doxorubicin treatment and transfection were used as negative control for apoptosis. After the DiOC6 treatment and following flow cytometric analyses, it is seen that the percentage of viable cells is very low (less than 5\%) in doxorubicin treated cells compared to the other three groups of cells. In the SC group, the percentage of viable cells is highest ( 90\%). In TAp63a-transfected cells, the percentage of dead cells is lower than the UC group, but is much higher than the SC group; and the percentage of viable cells is less than $60 \%$. In the TAp63a-LANA cotransfected cells, the percentage of viable cells is higher than the TAp63a-transfected cells $(\sim 70 \%)$.

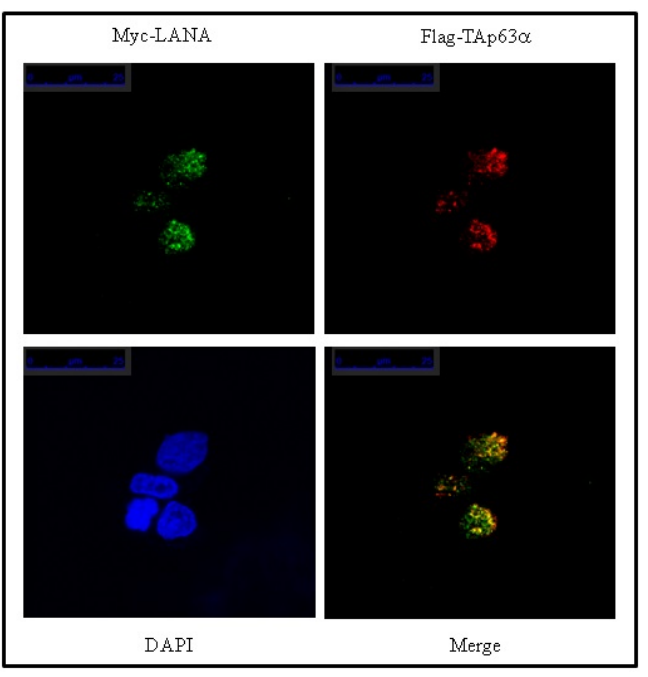

Figure 3: LANA co-localizes with p63 inside the nucleus. The nuclei are counterstained using DAPI (blue), Alexa Fluor 594 (red) is used for Flag-TAp63a and Alexa Fluor 488 (green) is used for MycLANA. The images were sequentially captured using confocal microscope and are merged, as required. Presence of both TAp63a (upper right panel) and LANA (upper left panel) in the nucleus is confirmed by comparing with DAPI staining (lower left panel). Colocalization of TAp63a and LANA are confirmed by the merged image (lower right panel) expressing a different color (yellow) along with the colors of Alexa Fluor 594 (red) and Alexa Fluor 488 (green). These results indicate an interaction between TAp63 $\alpha$ and LANA and thus a role of LANA for modulating the activities of TAp63a, thereby helping the cells escape apoptosis.

\section{Discussions}

\section{LANA helps cells escape apoptosis}

Doxorubicin treatment induces apoptosis in the treated cells by upregulation of various apoptotic molecules. It has also been shown previously that TAp63a plays a vital role in regulation of the cell cycle and apoptosis via upregulation of such apoptotic molecules in cells treated with chemotherapeutic agents like doxorubicin [10-13]. In our study it has been found that TAp63a-transfected cells show increased apoptosis compared to the control cells. Though the TAp63a-LANA transfected cells still shows a higher percentage of apoptosis compared to the control cells, interestingly, these cells show much less apoptosis compared to the cells transfected with TAp63a only. These results suggest that TAp63a induces apoptosis in doxorubicin-treated cells within 12 and 24 hours. These cells can escape apoptosis in the presence of LANA which interferes with the TAp63a-mediated cell cycle arrest by death receptor and/or mitochondrial pathways. 


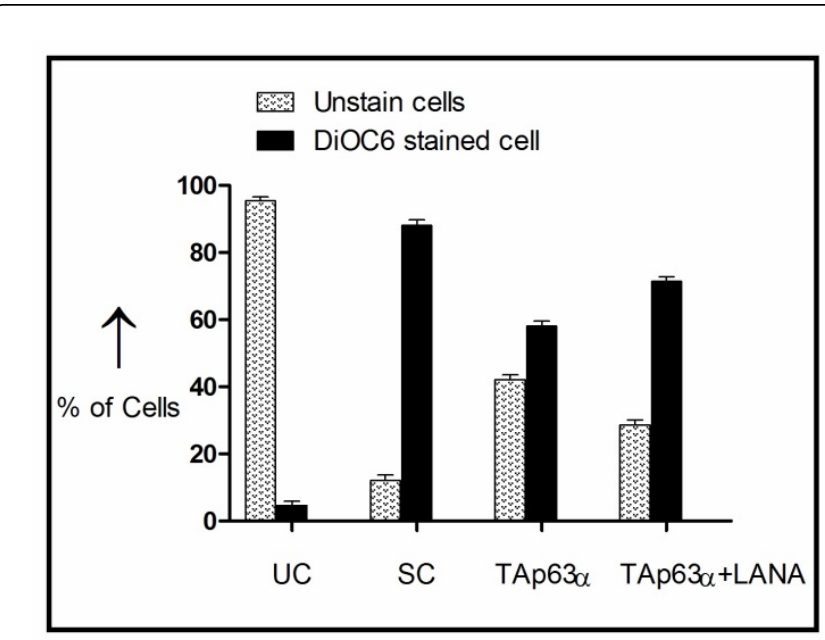

Figure 4: LANA reduces the TAP63a induced MMP loss. Cells tranfected with TAp63a alone and with both TAp63a and LANA were flow cytometrically assessed for reduction in MMP loss by DiOC6 fluorescence. The figure shows the representative results of three independent experiments. After the DiOC6 treatment, the percentage of viable cells is highest in doxorubicin-treated TAp63aLANA co-transfected cells compared to the other two groups of doxorubicin-treated cells. It suggests that the TAp63a-mediated loss of MMP may be reduced by the presence of LANA, indicating another mechanism to prevent apoptosis in cells. $\mathrm{p}<0.05$ was considered to reveal a significant difference.

\section{Formation of a stable complex between LANA and TAp63a results in decrease in apoptosis}

Earlier studies show that TAp63a-mediated apoptosis in doxorubicin-treated cells involves the upregulation of the CD95, TNF$\mathrm{R}$ and TRAIL- $\mathrm{R}$ death receptors. TAp63a also induces the mitochondrial apoptotic pathway by upregulation of the proapoptotic Bcl-2 family members like Bax and BCL2L11 and the expression of RAD9, DAP3 and APAF1 [30-32]. Our study demonstrates a reduction in apoptosis in doxorubicin-treated cells in the presence of LANA, suggesting a direct interaction between LANA and TAp63a in these stressed cells. To explore this, immunoprecipitation experiments were performed and the results show clear bands for both Myc-tagged LANA and Flag-tagged TAp63a transfected cells treated with appropriate antibodies (Figure 2). Interestingly, the banding patterns are similar in both the cells suggesting an interaction between TAp63a and LANA in the doxorubicin treated cells which have escaped apoptosis. Therefore it may be suggested that LANA alters the activities of TAp63a which upregulates the apoptotic proteins mentioned above and thus the cells can escape their programmed death caused by chemotherapeutic stress due to doxorubicin treatment.

\section{LANA co-localizes with p63 inside the nucleus to alter the activities of TAp63a}

Co-localization studies for TAp63a and LANA shows the presence of both these antigens in the nucleus of the doxorubicin-treated cells (Figure 3 upper panels and lower left panel). When the images for
TAp63 $\alpha$ and LANA are merged, presence of a different color (yellow) along with the colors of Alexa Fluor 594 (red) and Alexa Fluor 488 (green) is seen (Figure 3 lower right panel). This merged color helps us to infer that co-localization of these two antigens is seen and this colocalization results from a direct interaction between them. These results suggest a role of LANA for modulating the activities of TAp63a and retard the TAp63a-mediated upregulation of apoptotic molecules, thereby helping the cells escape apoptosis.

\section{Reduction in loss of mitochondrial membrane potential induced by TAp63a}

Previous studies report that TAp63a-mediated apoptosis results also form loss of mitochondrian membrane potential $(\triangle \Psi \mathrm{mMMP})$ [30-32]. It is seen in our study that after the DiOC6 treatment, the percentage of viable cells is highest in doxorubicin-treated TAp63aLANA cotransfected cells compared to the other two groups of doxorubicin-treated cells. All these three groups show less viable cells compared to the control Saos-2 cells (SC), as expected, because the killing effects of the chemotherapeutic agent are not fully reverted by LANA (Figure 4). These results suggest that the TAp63a-mediated apoptosis caused by loss of $\triangle \Psi$ mMMP may be reduced by the presence of LANA. LANA can interact with TAp63a and modulate its activities, as reported above, thereby indicating a reduction of loss of MMP as another mechanism to prevent apoptosis in cells.

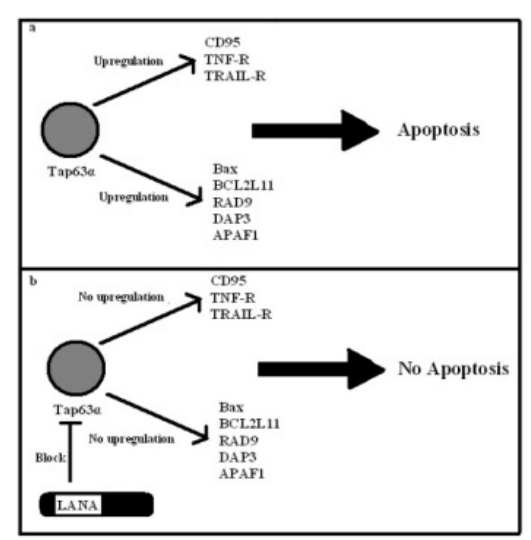

Figure 5: Model depicting inhibitory action of LANA against TAp63a-induced apoptosis. A) 9TAp63a upregulates various genes needed for apoptotic pathways when chemotherapeutic agents are present; and thus lead to apostosis. B) In KSHV infected cells, the presence of LANA alters the activities of TAp63a by interacting with it and leads to cell survival.

\section{Conclusion}

TAp63a and $\Delta \mathrm{Np} 63$, the major isoforms of plays various roles in development and cancers. Unlike its type-member of the family, the p53 tumor suppressor, the p63 can suppress as well as induce apoptosis depending upon other cellular conditions and treatment with chemotherapeutic agents. The TAp63a isoforms can induce apoptosis by inducing the expression of various death receptors and proteins for loss of mitochondrial membrane potential (Figure 5 panel a). As our study reports, it can interact directly with LANA, the latency-associated nuclear antigen of KSHV and therefore plays a major role in the survival of KSHV-infected cells (Figure 5 panel b). 
The results of the present study indicate a possible mechanism by which the infected cells can escape apoptosis and thus can survive in the diseased condition.

\section{Acknowledgments}

This work was supported by Department of Biotechnology, India sponsored project No: BT/PR/7044/MED/29/B55/2014. The funders had no role in study design, data collection and analysis, decision to publish, or preparation of the manuscript.

\section{References}

1. Chang Y, Cesarman E, Pessin MS, Lee F, Culpepper J, et al. (1994) Identification of herpesvirus-like DNA sequences in AIDS-associated Kaposi's sarcoma. Science 266: 1865-1869.

2. Cesarman E, Chang Y, Moore PS, Said JW, Knowles DM (1995) Kaposi's sarcoma-associated herpesvirus-like DNA sequences in AIDS-related body-cavity-based lymphomas. N Engl J Med 332: 1186-1191.

3. Soulier J, Grollet L, Oksenhendler E, Cacoub P, Cazals-Hatem D, et al. (1995) Kaposi's sarcoma-associated herpesvirus-like DNA sequences in multicentric Castleman's disease. Blood 86: 1276-1280.

4. Lu F, Tsai K, Chen HS, Wikramasinghe P, Davuluri RV, et al. (2012) Identification of host-chromosome binding sites and candidate gene targets for Kaposi's sarcoma-associated herpesvirus LANA. J Virol 86: 5752-5762.

5. Friborg J Jr, Kong W, Hottiger MO, Nabel GJ (1999) p53 inhibition by the LANA protein of KSHV protects against cell death. Nature 402 : 889-894.

6. Kaul R, Verma SC, Robertson ES (2007) Protein complexes associated with the Kaposi's sarcoma-associated herpesvirus-encoded LANA. Virology 364: 317-329.

7. Cotter MA 2nd, Robertson ES (1999) The latency-associated nuclear antigen tethers the Kaposi's sarcoma-associated herpesvirus genome to host chromosomes in body cavity-based lymphoma cells. Virology 264: 254-264.

8. De León Vázquez E, Kaye KM (2011) The internal Kaposi's sarcomaassociated herpesvirus LANA regions exert a critical role on episome persistence. J Virol 85: 7622-7633.

9. Barbera AJ, Chodaparambil JV, Kelley-Clarke B, Luger K, Kaye KM (2006) Kaposi's sarcoma-associated herpesvirus LANA hitches a ride on the chromosome. Cell Cycle 5: 1048-1052.

10. Murray-Zmijewski F, Lane DP, Bourdon JC (2006) p53/p63/p73 isoforms: an orchestra of isoforms to harmonise cell differentiation and response to stress. Cell Death Differ 13: 962-972.

11. Wu G, Nomoto S, Hoque MO, Dracheva T, Osada M, et al. (2003) DeltaNp63alpha and TAp63alpha regulate transcription of genes with distinct biological functions in cancer and development. Cancer Res 63: 2351-2357.

12. Yang A, Kaghad M, Wang Y, Gillett E, Fleming MD, et al. (1998) p63, a p53 homolog at 3q27-29, encodes multiple products with transactivating, death-inducing, and dominant-negative activities. Mol Cell 2: 305-316.

13. Crum CP, McKeon FD (2010) p63 in epithelial survival, germ cell surveillance, and neoplasia. Annu Rev Pathol 5: 349-371.

14. Levrero M, De Laurenzi V, Costanzo A, Gong J, Wang JY, et al. (2000) The p53/p63/p73 family of transcription factors: overlapping and distinct functions. J Cell Sci $113: 1661-1670$.
15. Oliner JD, Pietenpol JA, Thiagalingam S, Gyuris J, Kinzler KW, et al. (1993) Oncoprotein MDM2 conceals the activation domain of tumour suppressor p53. Nature 362: 857-860.

16. Haupt Y, Maya R, Kazaz A, Oren M (1997) Mdm2 promotes the rapid degradation of p53. Nature 387: 296-299.

17. Honda R, Tanaka H, Yasuda H (1997) Oncoprotein MDM2 is a ubiquitin ligase E3 for tumor suppressor p53. FEBS Lett 420: 25-27.

18. Kubbutat MH, Jones SN, Vousden KH (1997) Regulation of p53 stability by Mdm2. Nature 387: 299-303.

19. Calabrò V, Mansueto G, Parisi T, Vivo M, Calogero RA, et al. (2002) The human MDM2 oncoprotein increases the transcriptional activity and the protein level of the p53 homolog p63. J Biol Chem 277: 2674-2681.

20. Collavin L, Lunardi A, Del Sal G (2010) p53-family proteins and their regulators: hubs and spokes in tumor suppression. Cell Death Differ 17: 901-911.

21. Martin ME, Berk AJ (1998) Adenovirus E1B 55K represses p53 activation in vitro. J Virol 72: 3146-3154.

22. Ahuja D, Sáenz-Robles MT, Pipas JM (2005) SV40 large T antigen targets multiple cellular pathways to elicit cellular transformation. Oncogene 24: 7729-7745.

23. Pise-Masison CA, Brady JN (2005) Setting the stage for transformation: HTLV-1 Tax inhibition of p53 function. Front Biosci 10: 919-930.

24. Münger K, Scheffner M, Huibregtse JM, Howley PM (1992) Interactions of HPV E6 and E7 oncoproteins with tumour suppressor gene products. Cancer Surv 12: 197-217.

25. Teissier S, Ben Khalifa Y, Mori M, Pautier P, Desaintes C, et al. (2007) A new E6/P63 pathway, together with a strong E7/E2F mitotic pathway, modulates the transcriptome in cervical cancer cells. J Virol 81: 9368-9376.

26. Djelloul S, Tarunina M, Barnouin K, Mackay A, Jat PS (2002) Differential protein expression, DNA binding and interaction with SV40 large tumour antigen implicate the p63-family of proteins in replicative senescence. Oncogene 21: 981-989.

27. Santag S, Jäger W, Karsten CB, Kati S, Pietrek M, et al. (2013) Recruitment of the tumour suppressor protein p73 by Kaposi's Sarcoma Herpesvirus latent nuclear antigen contributes to the survival of primary effusion lymphoma cells. Oncogene 32: 3676-3685.

28. Petre CE, Sin SH, Dittmer DP (2007) Functional p53 signaling in Kaposi's sarcoma-associated herpesvirus lymphomas: implications for therapy. J Virol 81: 1912-1922.

29. Sarek G, Kurki S, Enbäck J, Iotzova G, Haas J, et al. (2007) Reactivation of the p53 pathway as a treatment modality for KSHV-induced lymphomas. J Clin Invest 117: 1019-1028.

30. Gressner O, Schilling T, Lorenz K, Schulze Schleithoff E, Koch A, et al. (2005) TAp63alpha induces apoptosis by activating signaling via death receptors and mitochondria. EMBO J 24: 2458-2471.

31. Soleimani R, Heytens E, Darzynkiewicz Z, Oktay K (2011) Mechanisms of chemotherapy-induced human ovarian aging: double strand DNA breaks and microvascular compromise. Aging (Albany NY) 3: 782-793.

32. Matin RN, Chikh A, Chong SL, Mesher D, Graf M, et al. (2013) p63 is an alternative 553 repressor in melanoma that confers chemoresistance and a poor prognosis. J Exp Med 210: 581-603.

33. Marchetti P, Castedo M, Susin SA, Zamzami N, Hirsch T, et al. (1996) Mitochondrial permeability transition is a central coordinating event of apoptosis. J Exp Med 184: 1155-1160. 